\title{
La construcción de indicadores de la actividad económica: una revisión bibliográfica
}

\author{
Indicators of Economic Activity: A Review
}

\author{
A elaboração de indicadores de atividade \\ econômica: uma revisão bibliográfica
}

\author{
Lya Paola Sierra-Suárez \\ Jaime Andrés Collazos-Rodríguez ** \\ Johana Sanabria-Dominguez \\ Pavel Vidal-Alejandro ${ }^{* * * *}$
}

DOI: https://doi.org/10.19053/01203053.v36.n64.2017.5132

Fecha de recepción: 11 de julio de 2016

Fecha de aceptación: 25 de mayo de 2017

\footnotetext{
* Doctora en Economía de la Universidad Autónoma de Madrid. Profesora asociada del Departamento de Economía de la Universidad Javeriana. Cali, Colombia. lyap@javerianacali.edu.co (iD) https://orcid.org/0000-0002-8909-8977

** Economista del Centro Regional de Estudios Económicos (CREE). Banco de la República. Cali, Colombia. jcollaro@banrep.gov.co iD https://orcid.org/0000-0002-3928-3242

*** Economista del Centro Regional de Estudios Económicos (CREE). Banco de la República. Cali, Colombia. jsanabdo@banrep.gov.co (iD) https://orcid.org/0000-0002-1868-1188

**** Doctor en Economía de la Universidad de La Habana. Profesor asociado del Departamento de Economía. Pontificia Universidad Javeriana. Cali, Colombia. pavel@javerianacali.edu.c

(iD https://orcid.org/0000-0001-8278-3122
} 


\section{Resumen}

Los indicadores de actividad económica son utilizados para medir el comportamiento de una economía cuando ningún otro tipo de indicador, como el producto interno bruto, puede proporcionar información sobre el estado de la economía de forma actualizada. En este documento se realiza una revisión de la literatura nacional e internacional sobre la construcción de indicadores de actividad económica. Adicionalmente, se ofrece un resumen de la metodología más utilizada en la construcción de índices de actividad económica, el modelo factorial dinámico (MFD) y sus diferentes tipos de estimación, resaltando las ventajas y desventajas. Finalmente, se presenta el método utilizado en la construcción del índice mensual de actividad económica para el Valle del Cauca (IMAE).

Palabras clave: indicador de actividad económica, modelo factorial dinámico.

Clasificación JEL: C52, C53, E3. 
Apuntes CENES Volumen 36, Número 64

julio - diciembre 2017. Págs. 79-107

\section{Abstract}

Economic indicators are used to measure the performance of the economy when other indicators, such as gross domestic product, may not provide information about the state of the economy in real time. This article provides a review of national and international literature about the construction of indicators of economic activity. Additionally, a summary of the methodology most commonly used in the construction of indices of economic activity, Factorial Dynamic Model (MFD) and different types of estimation is offered.

Keywords: index of economic activity, dynamic factor model. 


\section{Resumo}

Os indicadores econômicos são usados para medir o desempenho de uma economia quando nenhum outro tipo de indicador, como o Produto Interno Bruto, pode fornecer informações atualizadas sobre a situação econômica de um país. Este artigo apresenta uma revisão da literatura nacional e internacional sobre a elaboração de indicadores de atividade econômica. Adicionalmente, traz um resumo sobre a metodologia mais usada na sistematização de índices de atividade econômica, o Modelo Fatorial Dinâmico (MFD) e seus diferentes métodos de estimação.

Palavras-chave: Indicador de Atividade Econômica, Modelo Fatorial Dinâmico. 
Apuntes CENES Volumen 36, Número 64

julio - diciembre 2017. Págs. 79-107

\section{INTRODUCCIÓN}

En las últimas cuatro décadas, los indicadores sobre actividad económica se convirtieron en una herramienta muy utilizada por los agentes económicos alrededor del mundo para conocer de primera mano el estado actual de la economía y facilitar así la toma de decisiones. Esta tendencia generó que entidades estatales, privadas y académicas prestaran mayor interés al cálculo de dichos índices ante el importante rezago de las principales estadísticas económicas oficiales disponibles.

Existen diferentes tipos de indicadores de actividad económica, de los cuales los más desarrollados en el ámbito internacional han sido los coincidentes y líderes. Un índice de actividad econó- mica coincidente describe el ciclo de referencia o estado actual de la economía, mientras que un indicador líder busca dar señales de las tendencias futuras del ciclo económico. La razón de ser de los índices de actividad económica es que el producto interno bruto (PIB), indicador habitual para medir la actividad económica de un país o una región, se publica de forma trimestral o anual, y el rezago en su publicación es alto. Los indicadores coincidentes o líderes buscan construirse con una mayor frecuencia (mensual o semanal) y de esta manera proveer una evaluación más actualizada sobre el estado de la actividad económica. La información más oportuna que ofrecen los indicadores se convierte en una herramienta que contribuye a la toma de decisiones por parte de los diferentes agentes económicos. 
La experiencia acumulada en estos esfuerzos muestra que la construcción de indicadores de actividad económica parte de un conjunto amplio de variables macroeconómicas o financieras de alta frecuencia, que además de estar publicadas con poco nivel de rezago, coinciden con la información contenida en una variable referente a la economía (e.g., producto interno bruto) o de un sector específico (e.g., índice de producción industrial). Así, con esta información se identifican los puntos de giro o de inflexión de cada una de las series y se clasifican como coincidentes o líderes. En consecuencia, estas series son agregadas en un indicador sintético dependiendo de la categoría a la que pertenezcan. El objetivo final es resumir la mayor cantidad de información en un solo indicador o índice.

La motivación de este artículo proviene de la experiencia y de revisiones bibliográficas realizadas para la construcción de un indicador de actividad económica coincidente para el departamento del Valle del Cauca en Colombia. El indicador mensual de actividad económica (IMAE) ha sido el primero elaborado con la metodología de los MFD para una región de Colombia (Vidal, Sierra, Sanabria \& Collazos, 2015). Por tanto, esperamos que los contenidos metodológicos aquí resumidos puedan ayudar a replicar este ejercicio en otros departamentos del país. El IMAE se construye y publica desde el año 2015 por el Banco de la República y la Pontificia Universidad Javeriana, Cali.

Por lo anterior, este trabajo tiene como objetivo efectuar una revisión de la literatura nacional e internacional más relevante sobre la construcción de indicadores de actividad económica. Adicionalmente, se describe una de las metodologías más utilizadas para la construcción de este tipo de indicadores, los modelos factoriales dinámicos (MFD). Por ello, una de las contribuciones de este documento es describir formalmente los principales métodos de estimación para construir indicadores de actividad económica desde los MDF, resaltando sus ventajas y desventajas.

El artículo se encuentra organizado de la siguiente manera: en la segunda sección se presenta la definición de los indicadores económicos y su relación con la teoría de los ciclos económicos. En la tercera sección se describe formalmente uno de los modelos más utilizados en la construcción de índices de actividad económica, los MFD, sus principales métodos de estimación, y se efectúa una revisión bibliográfica sobre los principales indicadores de actividad económica en el orden mundial que utilizan dichas técnicas. En la cuarta sección se analizan los estudios realizados sobre el tema en Colombia. A continuación, en la quinta sección, se presenta una aplicación de los MFD 
en la elaboración de un indicador de actividad económica regional. Específicamente se incluyen los pasos llevados a cabo en la construcción y estimación trimestral de la última versión del índice de actividad económica para el Valle del Cauca, IMAE. Por último, se exponen los comentarios finales.

\section{EL CICLO ECONÓMICO Y LOS INDICADORES DE ACTI- VIDAD ECONÓMICA}

La teoría de los ciclos es el sustento teórico para las distintas metodologías de construcción de indicadores de actividad económica (Nieto \& Melo, 2001). La obra de Burns y Mitchell (1946) define los ciclos económicos como fenómenos recurrentes en la actividad económica, descritos por periodos de fluctuaciones de la actividad económica agregada. Los ciclos se caracterizan por aparecer simultáneamente en diferentes sectores de la economía en sus distintas fases: expansión, contracción, recesión y recuperación, secuencias que son recurrentes pero no periódicas. En cuanto a su duración, los ciclos varían entre un año y diez o doce años (Burns \& Mitchell, 1946).

Puesto que el ciclo económico se ocupa de las fluctuaciones de la economía agregada y no se encuentra explicado por una sola causa, los indicadores cíclicos -construidos como la agrega- ción de un conjunto de variables- se articulan muy bien a esta teoría. En efecto, existen grupos de variables mensuales y trimestrales para monitorear el desempeño de los diferentes sectores económicos, pero ellos ofrecen señales dispersas $y$, en muchos casos, contradictorias sobre el estado actual de la economía. Además, en el ámbito individual, las series pueden presentar inconvenientes debido a que su comportamiento puede variar por errores de medición o cambios estructurales del sector que no representen las fluctuaciones del ciclo (agregado).

Ante estos inconvenientes, la solución ha sido la creación de índices compuestos que combinen las diferentes series. Con los índices se busca aumentar la probabilidad de obtener las señales verdaderas sobre el ciclo y disminuir las falsas. En general, la idea es que los comovimientos observados entre un conjunto amplio de variables económicas a lo largo del ciclo permiten reflejar los patrones de la dinámica común de un grupo de datos y, por lo tanto, proporcionan una señal más clara y convincente del ciclo de una economía, lo cual es más efectivo que analizar cada una de las variables por separado.

La construcción del indicador busca encontrar secuencias repetitivas y comunes en las series para usarlas en la identificación y pronóstico del ciclo económico. Al respecto, se puede ha- 
cer una distinción de tres tipos de indicadores: coincidentes, rezagados y adelantados. El indicador coincidente estima la evolución contemporánea de la actividad económica. El indicador adelantado presenta un comportamiento anticipado respecto al ciclo económico de referencia. Por su parte, el indicador rezagado valida el comportamiento de la actividad económica en meses posteriores.

Los primeros ejercicios sobre estos indicadores fueron desarrollados por Burns y Mitchell (1946), quienes diseñaron índices por categorías, agregando las series que tuvieran similar comportamiento a la serie de referencia. Estas series, a su vez, eran clasificadas como líderes, coincidentes o rezagadas; de esta selección dependía la categoría del índice calculado. A partir de esta metodología se elaboraron otros índices compuestos desde el NBER ${ }^{1}$, para la $\mathrm{OECD}^{2}$ y en la Unión Europea; la mayoría fue construida con base en el comportamiento de una serie de referencia de la economía o de un sector específico. Para tal fin, se analizaban estadísticas descriptivas, se desestacionalizaban y se aislaba el componente estrictamente cíclico; además, en el proceso se identificaban sus puntos de quiebre, picos, valles hasta fases de expansión y contracción.
Posteriormente, tales estadísticas descriptivas se clasificaban como líderes, coincidentes y rezagadas para luego agregarlas en un índice compuesto de acuerdo con cada categoría.

Con el paso de las décadas fueron apareciendo en la literatura indicadores coincidentes y líderes construidos con una estructura estadística más formal, en su mayoría conocidos como modelos factoriales dinámicos -MFD-, y definidos como técnicas de reducción de la dimensión de un conjunto amplio de variables a través de análisis factorial. Generalmente, estos modelos parten del supuesto de que hay una variable no observada o latente, como el estado de la economía, que es común a las diferentes variables observadas, como las variables mensuales económicas, y que puede ser estimada. Es decir, a partir de los comovimientos de las diferentes variables agregadas se obtiene un índice, una estimación del valor de la variable no observable, que es la que aproxima el ciclo de la economía en tiempo real o futuro. Existen varias técnicas para calcular los MFD, y su uso depende de la manera como se extraen los factores y cómo se calcula el componente común. Más adelante en el documento se hablará de cada una de estas técnicas.

\footnotetext{
1 The National Bureau of Economic Research. Dicha institución se encarga, entre otras cosas, de anunciar la ocurrencia de las distintas fases de los ciclos de negocios, mostrar las características de la economía a lo largo de los mismos y justificar los anuncios (Arango, Arias, Flórez \& Jalil, 2008).

2 Organización Económica para el Comercio y el Desarrollo.
} 
Apuntes CENES Volumen 36, Número 64

julio - diciembre 2017. Págs. 79-107

LOS MODELOS FACTORIALES DINÁMICOS Y ALGUNAS DE SUS TÉCNICAS DE ESTIMACIÓN

La construcción de indicadores sintéticos de actividad económica se ha convertido en una herramienta de uso extendido en la valoración de la coyuntura económica en diferentes países, especialmente en los bancos centrales, siendo los referentes metodológicos de mayor uso los índices que se han construido en Estados Unidos (i.e., el Chicago FED -CFNAI-, que estima de manera coincidente la actividad económica mensual de Estados Unidos y el índice de las condiciones reales de negocios de Aruoba-Diebold-Scotti publicado por la FED de Philadelphia) y Europa (i.e., el indicador EuroCOIN del Banco de Italia, que mide la actividad económica de la zona euro).

La mayoría de los anteriores ejercicios se basó principalmente en metodologías derivadas de los modelos factoriales dinámicos (MFD), construidos en entornos ricos en datos, es decir, con una gran cantidad de series económicas, o a partir de pocas variables. Existen varias técnicas para calcular los MFD y su uso depende de la manera como se extraen los factores y cómo se calcula el componente común. Entre las principales aproximaciones que han aparecido en la literatura para estimar los factores dinámicos latentes sobresalen el método de componentes principales (CP), el filtro de Kalman (FK) y los métodos híbridos (MH).

El modelo factorial dinámico asume que una matriz $Y$, de $\mathrm{N}$ variables de actividad económica observadas, puede ser representado como la suma de dos componentes no observables que son mutuamente independientes: un o unos componentes comunes a todas las variables $\left(F_{t}\right)$,, en las aplicaciones relacionadas con indicadores de actividad económica, solo se toma el primer factor, que representa el estado de la economía, y uno idiosincrático $\left(\mu_{t}\right)$, el cual representa la dinámica propia de cada serie. De acuerdo con lo anterior, la ecuación para las series observadas, $Y$, se puede representar en forma vectorial como:

$$
Y_{t}=\mathrm{P} F_{t}+\mu_{t}
$$

en donde $\mathrm{Y}_{t}=\left(y_{1 \imath} y_{2 t} \ldots y_{N T}\right)^{\prime}$ contiene series mensuales de actividad económica; $F_{t}=\left(F_{1}, \ldots, F_{T}\right)^{\prime}$ representa choques comunes; representa la carga o peso que tienen cada uno de los factores o choques, y $\mu_{t}=\left(\mu_{1}, \ldots, \mu_{N}\right)^{\prime}$, que corresponde a los componentes específicos o idiosincráticos.

La dinámica de los factores viene dada por:

$$
\Phi(\mathrm{B}) f_{t}=a_{t}
$$

donde $a_{t}$ es ruido blanco normal multivariante con matriz de varianzas y co- 
varianzas $\sum a .$. , siendo $B$ el operador de rezagos. Los componentes idiosincráticos también pueden presentar estructura dinámica de la forma:

$$
\Phi(\mathrm{B})=I-\Phi_{1} B-\ldots-\Phi_{p} \mathrm{~B}^{p},
$$

donde $D(B)=\operatorname{diag}\left(D_{i}(B)\right)$ es una matriz diagonal que recoge la dinámica específica de cada perturbación idiosincrásica; $\quad D_{i}(B)=1-d_{i 1} B-\ldots-d_{i p_{i}} B^{p_{i}}$, $\mathrm{i}=1,2, \ldots, \mathrm{m}$, corresponde a la estructura autorregresiva del componente en cada serie representada con el operador de rezagos; $e_{t}$ es un ruido blanco de media cero con matriz de covarianzas diagonal.

Como se mencionó al inicio de esta sección, las técnicas más utilizadas en el estado de la cuestión para estimar el componente común de los MFD son: estimación mediante componentes principales, CP (la cual se centra en explotar la dimensión cruzada $\mathrm{N}$ ), estimación mediante el filtro de Kalman, FK (la cual se centra en explotar la dinámica tanto del componente común, como del específico) y los modelos híbridos que contemplan la utilización de ambos tipos de estimación, CP y FK. Para un análisis más detallado de los MFD véanse Stock y Watson (2011).
Los CP se constituyen en la técnica más utilizada en el estado de la cuestión, no solo para la construcción de indicadores de actividad económica, sino en temas de competitividad y otros subsectores ${ }^{3}$. Esta herramienta permite sintetizar información bajo entornos ricos en datos a través del cálculo de factores o grupos. El método determina el menor número de componentes principales que expliquen la mayor proporción de la variabilidad del conjunto original de datos, logrando así una reducción del problema estudiado con una pequeña pérdida de información. El proceso de reducción de un conjunto amplio de variables en factores permite que estos grupos estén altamente relacionados con las variables seleccionadas inicialmente. Los trabajos de mayor referencia internacional para la aplicación de dicha metodología en el diseño de indicadores de actividad económica son los de Stock y Watson (2002) y Forni et al. (2000 y 2005), los cuales realizaron varias propuestas basadas en componentes principales, como los CP estáticos, dinámicos y ponderados. La ventaja de la estimación del indicador de actividad económica mediante $\mathrm{CP}$ radica en que es una técnica de estimación relativamente sencilla en la práctica. La desventaja está

\footnotetext{
${ }^{3}$ Los componentes principales, como métodos de estimación de los MFD, también han sido utilizados en otras aplicaciones, como en las finanzas o a las materias primas, por ejemplo. Este último es el caso del estudio de Poncela, Senra y Sierra (2014), quienes evaluaron el comovimiento existente en 44 series de materias primas no energéticas.
} 
en la necesidad de un número grande de variables económicas y tamaño de muestra, para asegurar la consistencia del indicador ${ }^{4}$.

Por otra parte, el filtro tiene su origen en el documento de Kalman (1960). Es el principal algoritmo para estimar sistemas dinámicos representados en la forma estado-espacio. La estimación del componente común a través del filtro de Kalman tiene la ventaja de que modela la dinámica de los componentes específicos (ecuación 3), por lo que permite realizar predicciones más precisas del estado actual de la economía, o nowcasting. Igualmente, permite el uso de irregularidades en los datos (frecuencias mixtas, datos faltantes). Sin embargo, no admite trabajar con muchas series, pues la dimensión del vector de estado podría ser un problema. La estimación del MFD se puede hacer por máxima verosimilitud aplicando el filtro de Kalman. En Hamilton (1994), y Koopman, Shephard y Doornik (1999) se encuentra una descripción completa del filtro de Kalman.

Los métodos híbridos combinan los anteriores métodos de estimación, componentes principales y filtro de Kalman, con el fin de aprovechar apropiadamente las ventajas de ambos tipos de estimación. El artículo seminal
Giannone, Reichlin, y Small (2008), describe la metodología que luego fue formalizada en Doz, Giannone, y Reichlin (2011). Los autores utilizan ambos tipos de estimación en dos pasos: en el primero se extraen los factores comunes mediante $\mathrm{CP}$ y en el segundo se utilizan las condiciones iniciales dadas por CP para la estimación mediante KF. Este método es especialmente útil en la medida en que puede resolver problemas de datos omitidos en una gran base de datos.

Las aplicaciones recientes del MFD incorporan la idea de que el comportamiento dinámico de la actividad económica depende del estado de la economía. Por ejemplo, las expansiones y contracciones del ciclo económico, que serían dos estados de la economía, pueden ser tratadas con diferentes objetos probabilísticos. Este tipo de modelos, desarrollados inicialmente por Kim y Yoo (1995) y Diebold y Rudebush (1996), combinan el enfoque basado en MFD, el cual captura el comovimiento existente en variables económicas, y los regímenes cambiantes estocásticos, que capturan los cambios de regímenes observados en la dinámica de estas variables. Los llamados modelos de regímenes cambiantes estocásticos (Markov switching regimes) son especialmente útiles para

${ }^{4}$ Poncela y Ruiz (2012) evaluaron que el número mínimo de series para asegurar consistencia en el estimador de CP es 30 . 
hallar los puntos de inflexión de una economía (probabilidad de entrar en recesión, en periodos de desaceleración de la economía, o probabilidad de entrar en periodo de recuperación). Para las autoridades económicas esta información puede ser importante para tomar medidas anticíclicas adecuadas.
En la Tabla 1 se presentan los artículos revisados, relacionados con cada una de estas técnicas de medición. En la tabla también se evidencia el objetivo de la aplicación que se realiza en cada artículo, ya sea para la identificación del ciclo, o los puntos de inflexión del ciclo económico, o para el pronóstico del mismo.

Tabla 1. Trabajos sobre indicadores de actividad económica que utilizan métodos de estimación basados en MFD

\begin{tabular}{|c|c|c|c|}
\hline Referencia & Aplicación & $\begin{array}{l}\text { Número de } \\
\text { variables } \\
\text { utilizadas }\end{array}$ & $\begin{array}{c}\text { Técnica de estimación } \\
\text { del MFD }\end{array}$ \\
\hline $\begin{array}{l}\text { Chicago Fed National } \\
\text { Activity Index (CFNAI) }\end{array}$ & $\begin{array}{l}\text { Indicador de actividad eco- } \\
\text { nómica (CFNAI) para Estados } \\
\text { Unidos }\end{array}$ & 85 & $\begin{array}{l}\text { Componentes princi- } \\
\text { pales }\end{array}$ \\
\hline $\begin{array}{l}\text { Center for Econom- } \\
\text { ic Policy } \\
\text { (CEPR) }\end{array}$ & $\begin{array}{l}\text { Indicador de actividad econó- } \\
\text { mica (EuroCOIN) }\end{array}$ & 1000 & $\begin{array}{l}\text { Componentes princi- } \\
\text { pales }\end{array}$ \\
\hline $\begin{array}{l}\text { Forni, Hallin, Lippi } \\
\text { y Reichlin (2001) }\end{array}$ & $\begin{array}{l}\text { Indicador de actividad econó- } \\
\text { mica para la zona euro }\end{array}$ & 89 & $\begin{array}{l}\text { Componentes princi- } \\
\text { pales dinámicos }\end{array}$ \\
\hline Stock y Watson (2002) & $\begin{array}{l}\text { Predicción del PIB de Estados } \\
\text { Unidos }\end{array}$ & 149 & $\begin{array}{l}\text { Componentes princi- } \\
\text { pales }\end{array}$ \\
\hline Schumacher (2007) & Predicción del PIB de Alemania & 124 & $\begin{array}{l}\text { Componentes princi- } \\
\text { pales }\end{array}$ \\
\hline $\begin{array}{l}\text { Aruoba, Diebold y Sco- } \\
\text { tti (2009) }\end{array}$ & $\begin{array}{l}\text { Indicador de actividad econó- } \\
\text { mica semanal para Estados } \\
\text { Unidos }\end{array}$ & 5 & Filtro de Kalman \\
\hline $\begin{array}{l}\text { Stock y Watson (1989, } \\
\text { 1991) }\end{array}$ & $\begin{array}{l}\text { Indicador coincidente, líder y } \\
\text { de recesión para Estados Uni- } \\
\text { dos }\end{array}$ & $5-7$ & Filtro de Kalman \\
\hline $\begin{array}{l}\text { Diebold y } \\
\text { Rudebush (1996) }\end{array}$ & $\begin{array}{l}\text { Indicador de actividad eco- } \\
\text { nómica para Estados Unidos, } \\
\text { con enfoque en los puntos } \\
\text { de inflexión del ciclo (Turning } \\
\text { points). }\end{array}$ & 4 & $\begin{array}{l}\text { MFD con regímenes } \\
\text { cambiantes estocás- } \\
\text { ticos "Markov swit- } \\
\text { ching regimes" }\end{array}$ \\
\hline
\end{tabular}


Apuntes CENES Volumen 36, Número 64

julio - diciembre 2017. Págs. 79-107

\begin{tabular}{|l|l|c|l|}
\hline $\begin{array}{l}\text { Angelini, Banbura } \\
\text { y Rünstler (2008) }\end{array}$ & $\begin{array}{l}\text { Predicción de la actividad eco- } \\
\text { nómica zona euro }\end{array}$ & 85 & $\begin{array}{l}\text { Filtro de Kalman (Kal- } \\
\text { man Smoother) }\end{array}$ \\
\hline $\begin{array}{l}\text { Camacho y Doménech } \\
\text { (2012) }\end{array}$ & $\begin{array}{l}\text { Indicador de actividad econó- } \\
\text { mica mensual para España, } \\
\text { predicción de PIB de España }\end{array}$ & 12 & Filtro de Kalman \\
\hline $\begin{array}{l}\text { Camacho y Pérez- } \\
\text { Quirós (2010) }\end{array}$ & Predicción de la zona euro & 13 & Filtro de Kalman \\
\hline $\begin{array}{l}\text { Camacho, Pérez- } \\
\text { Quirós y Poncela } \\
\text { (2014) }\end{array}$ & $\begin{array}{l}\text { Predicción de los puntos de in- } \\
\text { flexión de la zona euro }\end{array}$ & 13 & $\begin{array}{l}\text { MFD con regímenes } \\
\text { cambiantes estocás- } \\
\text { ticos "Markov swit- } \\
\text { ching regimes" }\end{array}$ \\
\hline $\begin{array}{l}\text { Doz, Giannone y } \\
\text { Rei-chlin (2011) }\end{array}$ & $\begin{array}{l}\text { Predicción de la actividad eco- } \\
\text { nómica zona euro }\end{array}$ & 85 & $\begin{array}{l}\text { Método híbrido: esti- } \\
\text { mación en dos pasos } \\
\text { (CP y KF) }\end{array}$ \\
\hline
\end{tabular}

Es importante resaltar que no solo se han realizado indicadores de actividad económica para países o regiones, también se han estimado indicadores tendientes a monitorear la actividad económica global. Algunos trabajos en esta línea son los de Golinelli y París (2014); Drechsel, Giesen y Lindner (2014); Ferrara y Marsilli (2014); y Camacho y Martínez-Martin (2015). En el trabajo de Golinelli y Parigi (2014) se combinaron aproximadamente 7.000 series económicas de países avanzados y emergentes para obtener predicciones trimestrales del PIB y del comercio mundial. Drechsel et al. (2014) encontraron que varios indicadores globales líderes mensuales mejoraron las previsiones en tiempo real del PIB mundial del Fondo Monetario Internacional -FMI-. En el mismo sentido, Ferrara y Marsilli (2014) desarrollaron un MFD para resumir la información de una base de datos mensual de gran tamaño en un pequeño número de factores y lo utilizaron en un marco MIDAS (Mixed-data sampling, por sus siglas en inglés). Al igual que el artículo anterior, las previsiones del PIB mundial de este modelo superaron las del FMI. Por su lado, Camacho y Martínez-Martin (2015) desarrollaron un MFD basado en regímenes cambiantes estocásticos (Markov switching) para computar inferencias sobre las fases del ciclo económico mundial.

Aunque el análisis factorial dinámico, con sus diferentes variantes o métodos de estimación, ha sido la metodología más utilizada en la estimación de indicadores de actividad económica en lo últimos años, se han construido otros indicadores como es el caso del trabajo de Choi y Varian (2011), quienes recurrieron a las tendencias de búsqueda en Google, e información de Federal Express y Master Card en la web, en- 
tre otras, para construir indicadores de actividad económica en tiempo real. Los autores sugirieron que información sobre las ventas de automóviles y sus partes, las solicitudes de beneficio por desempleo, los planes de viajes y la confianza del consumidor son útiles a la hora de predecir la actividad económica actual, o el llamado nowcasting ${ }^{5}$. Estos encuentran que los modelos autorregresivos (AR) con ajuste estacional que incluyen variables de Google Trends, tienden a superar entre $5 \%$ y $20 \%$ a los modelos que excluyen estos predictores.

\section{INDICADORES DE ACTIVI- DAD ECONÓMICA EN CO- LOMBIA}

En la literatura colombiana, el desarrollo de indicadores de actividad económica data desde los noventa. Los primeros ejercicios trataron, en su mayoría, de calcular el ciclo del PIB y sus puntos de giro. En efecto, Maurer y Uribe (1996) calcularon el ciclo de referencia mediante un sistema de indicadores cíclicos para anticipar los puntos de giro del ciclo económico colombiano con la metodología de NBER- OCDE; Ripoll, Misas y López (1995) estimaron y desarrollaron una de las primeras caracterizaciones del ciclo de la industria colombiana utilizando la experiencia de los analistas de coyuntura económica y modelos lineales como los polinomios de Almon y los análisis impulso respuesta; Salazar (1996) desarrolló un índice coincidente y adelantado a partir de variables referentes a la economía nacional;

Igualmente, en la década del 2000 aparecieron trabajos similares que, en general, consistieron en identificar el ciclo de una variable de referencia de la economía colombiana con sus puntos de inflexión, tal como lo resaltó Marcillo (2013). Los trabajos con estas características son los de Avella y Fergusson (2004); Arango y Melo (2006) Arango, Arias, Flórez y Jalil (2008); y Alfonso, Arango, Arias, Cangrejo y Pulido (2012).

A su vez, Nieto y Melo (2001) elaboraron un índice coincidente a través de una modificación del modelo desarrollado por Stock y Watson $(1989,1991)$. Con lo anterior, se buscó que las variables coincidentes estuvieran cointegradas y se involucrara así la estacionalidad dentro del modelo. Melo, Nieto, Posada, Betancourt y Barón (2001) generaron un indicador mensual coincidente para la economía colombiana utilizando la metodología

\footnotetext{
${ }_{5}^{5}$ Nowcasting es un término utilizado en literatura de indicadores de actividad económica para describir la predicción del pasado reciente, el presente y el futuro cercano, teniendo en cuenta que no se encuentra información sobre la misma.
} 
modificada de Stock y Watson desarrollada en Melo y Nieto (2001). Este trabajo correspondió a la primera parte de un proyecto de indicadores para la economía colombiana, en el cual se pretendió estimar un índice líder, plasmado luego en Melo, Nieto y Ramos (2003). A su vez, Castro (2003) construyó índices rezagados, coincidentes y adelantados para la actividad económica colombiana y caracterizó los ciclos de los negocios; para su trabajo utilizó el MFD de Forni, Lippi, Hallin, Reichlin, (2000). Por otro lado, Rozo (2008) planteó otro indicador líder (predicción del PIB colombiano) utilizando la técnica de pronósticos combinados propuesta por Stock y Watson (2004).

Por su parte, Kamil, Pulido y Torres (2010) construyeron un indicador mensual líder de la actividad económica en Colombia (IMACO) a través de un modelo factorial estimado por componentes principales. Por último, Marcillo (2013) construyó un indicador líder para identificar y comprobar la existencia de variables que anteceden el comportamiento del PIB a través del uso de un MFD aplicado por Forni et al. (2002).

Finalmente, dentro de los trabajos más recientes publicados en la evidencia nacional sobresalieron los indicadores sectoriales adelantados de actividad -ISAAC- para Colombia, elaborados por Mejía, Monsalve, Parra, Pulido y Reyes (2013), y el indicador de seguimiento a la economía -ISE- elaborado por el Departamento Administrativo Nacional de Estadística (DANE); ambos indicadores se fundamentaron en técnicas distintas a los MFD.

En efecto, Mejía et al. (2013) aplicaron la metodología de pronóstico (nowcast) a corto plazo de series económicas diseñada por Choi y Varian (2011), para así construir los indicadores sectoriales adelantados de actividad -ISAAC-. En sí, este trabajo consistió en extraer estadísticas a partir de la frecuencia de búsqueda en internet de determinadas palabras, mediante la utilización de Google Trends (GT). Estos indicadores mensuales permitieron anticipar la tendencia del PIB de las nueve grandes ramas de actividad y el consolidado nacional a corto plazo. Por eso, mediante la agregación de los distintos ISAAC se construyó el indicador ISAAC + buscando reflejar la tendencia de crecimiento del PIB total.

Mientras tanto, el DANE publica mensualmente el ISE, un índice sintético que mide la evolución de la actividad económica a corto plazo. Este ejercicio combina un pequeño conjunto de variables representativas de las actividades agropecuaria, industrial y de servicios. Los resultados del indicador se ajustan a la metodología de las cuentas nacionales trimestrales 
aplicando el método proporcional de Denton. El ISE permite identificar la estacionalidad, la tendencia y el ciclo de la dinámica productiva a lo largo del tiempo (Nota técnica: Indicador de Seguimiento a la Economía -ISE, 2014).

En el ámbito departamental se encuentran los trabajos de Alonso (2006) y Gómez; Sarmiento y Fajardo (2016) para la economía caucana. El primero estimó un MFD generalizado para el periodo 1960-2002, empleando el modelo de Forni, Hallin, Lippi y Reichlin (2003), y los segundos construyen un indicador líder a corto y largo plazo a partir de CP, FK y la metodología NBER. Por otra parte, Vidal et al. (2015) construyeron un indicador de actividad económica mensual para el Valle del Cauca -IMAE-, que estimó de manera coincidente la evolución de la actividad económica del departamento. En la siguiente sección se presentan la segunda versión del indicador mencionado anteriormente, el cual incorpora un número mayor de variables, y los pasos que se siguieron para la construcción del mismo. Se espera que la siguiente sección y los contenidos metodológicos presentados anteriormente permitan ayudar a replicar este ejercicio en otros departamentos del país.

\section{ESTIMACIÓN DE UN INDICA- DOR DE ACTIVIDAD ECONÓ- MICA DEPARTAMENTAL}

Desde el año 2015, el departamento del Valle del Cauca cuenta con el indicador mensual de actividad económica (IMAE), el cual se viene actualizando trimestralmente, y se da a conocer a la comunidad regional a través de un boletín que publica la Universidad Javeriana en su sitio $w^{6} b^{6}$, y además, forma parte de los contenidos del Boletín Económico Regional (BER) del suroccidente colombiano del Banco de la República. La segunda versión del indicador, la cual incorpora un número mayor de variables, empieza a estimarse a partir del cuarto semestre de 2016, una vez se ha hecho la retroalimentación con la región y una evaluación de variables adicionales. Dado que el DANE solo calcula para la región el PIB con frecuencia anual y lo da a conocer con rezago de diez meses, el IMAE se ha venido convirtiendo en una herramienta de interés para empresarios, entidades públicas y la comunidad académica, pues permite conocer casi en tiempo real la situación de la actividad económica y el punto en que se encuentra en relación con el ciclo económico y en comparación con el total nacional.

\footnotetext{
${ }^{6}$ http://imae.javerianacali.edu.co
} 
Para las primeras estimaciones del IMAE, el esfuerzo inicial consistió en organizar en el departamento el catastro de variables con frecuencia mensual que se relacionaran con la actividad económica. Inicialmente se contó con una base de datos de más de veintisiete variables, de las cuales solo se seleccionaron siete en la primera versión del IMAE (para un análisis más detallado de la metodología ver Vidal, et. al. 2015), y doce en la segunda versión (las cuales se presen-tan en esta sección). Para la selección de las variables se tuvo en cuenta que exhibieran una alta correlación anual con el PIB departamental, que repre- sentaran diferentes sectores o componentes de demanda de la economía de la región, y que fuera posible disponer cada trimestre de su actualización con poco tiempo de rezago.

En la Tabla 1 del apéndice se presenta una breve descripción de las variables que se añadieron en la segunda versión del indicador, y su importancia económica.

Las variables que actualmente se consideran en la estimación del IMAE se relacionan en la Tabla 2. Allí, además, resumimos la información económica contenida en cada variable.

\section{Tabla 2. Variables contenidas en el IMAE*}

\begin{tabular}{|l|l|}
\hline Variable & Información que contiene \\
\hline Caña molida (Valle) & Agricultura \\
\hline Despachos de cemento (Valle) & Construcción y vivienda \\
\hline $\begin{array}{l}\text { Consumo de energía del sector no } \\
\text { residencial (Valle) }\end{array}$ & $\begin{array}{l}\text { Actividad empresarial en general (incluyendo comercio y } \\
\text { servicios) }\end{array}$ \\
\hline Venta de vehículos nuevos (Valle) & Ingreso de los hogares, consumo, condiciones de crédito \\
\hline Exportaciones (Valle) & Actividad empresarial \\
\hline $\begin{array}{l}\text { IPIR: índice de producción industrial } \\
\text { regional (Valle) }\end{array}$ & Industria \\
\hline Importaciones (Valle) & Actividad empresarial, comercio, consumo \\
\hline Índice de confianza del consumidor* & Consumo \\
\hline Índice de condiciones económicas* & Consumo \\
\hline $\begin{array}{l}\text { Registro de los peajes del transporte } \\
\text { de carga* }\end{array}$ & Transporte, industria y comercio \\
\hline Volumen corrugado* & Industria y comercio \\
\hline Cartera de crédito bruta* & Sector financiero, consumo, actividad empresarial \\
\hline
\end{tabular}

*Variables que se añadieron en la segunda versión del IMAE. 
Las variables seleccionadas contienen información directa o indirecta, sobre diferentes actividades claves de la economía regional, en cuanto a la oferta o la demanda, o ambas. Se aprecia que ellas reflejan eventos más allá del propio sector donde se contabilizan, y que de manera repetitiva se refieren a un mismo sector o agregado económico. Esto no resulta en un problema, sino en una virtud, pues el IMAE no es una suma simple de valores, sino que resulta de encontrar el comovimiento entre las series, el cual representa el estado actual de la economía del Valle.

Una vez que se seleccionan las variables, estas se transforman: se desestacionalizan, se diferencian según el orden de integración de cada una, y finalmente se estandarizan (ver Tabla 2 en el apéndice, para detallar el tratamiento realizado a cada serie). Luego se aplica una metodología de tres pasos, la cual puede examinarse en detalle en Vidal et al. (2015). En resumen, los pasos son los siguientes:

Paso 1: se emplea la metodología pura de Stock y Watson (1991). Mediante un modelo factorial dinámico, se estima con el filtro de Kalman un factor común a todas las doce series. El factor depende de la estimación de unos pesos (ponderaciones o matriz de cargas de la ecuación 1) que se actualizan trimestralmente. Los últimos pesos estimados se relacionan en la Tabla 3.

Tabla 3. Pesos de las variables dentro del IMAE

\begin{tabular}{|l|c|}
\hline & Pesos (en la matriz de cargas) \\
\hline Caña molida & 0.03 \\
\hline Despachos de cemento & 0.07 \\
\hline Volumen de corrugado & 0.04 \\
\hline Índice de producción industrial regional & 0.21 \\
\hline Peajes de carga & 0.06 \\
\hline Exportaciones & 0.18 \\
\hline Importaciones & 0.13 \\
\hline Venta de vehículos nuevos & 0.08 \\
\hline Índice de confianza del consumidor & 0.07 \\
\hline Índices de condiciones económicas & 0.11 \\
\hline Cartera de crédito bruta & 0.001 \\
\hline Consumo de energía del sector no residencial & 0.03 \\
\hline
\end{tabular}

Paso 2: se aplica el método de Litterman (1983) para mensualizar el PIB anual del DANE para el departamen- to, a partir del factor común estimado en el paso 1. Esto permite incorporar al indicador la información sobre los 
datos anuales del PIB departamental hasta donde están publicados por el DANE. Con esto se persigue que los ciclos del indicador tengan mayor coherencia con las tasas anuales de crecimiento del PIB.

Paso 3: se aplica la metodología de los modelos estructurales univariantes de Harvey (1989), que posibilita suavizar la trayectoria del indicador a partir de separar el ciclo del resto de los componentes de la serie. Se obtiene así una trayectoria cíclica del IMAE sobre una media cero. Cuando el IMAE se en- cuentra por encima (debajo) de cero, esto refleja que la actividad económica está creciendo por encima (debajo) de su media histórica, la cual era de $4 \%$ en el período 2001-2015.

Así, se aprecia en la Figura 1 la coincidencia entre los datos anuales del PIB del DANE y la trayectoria mensual del IMAE, hasta el 2015. Para el año 2016 y el primer trimestre del 2017, las estimaciones del crecimiento económico a través del IMAE han reflejado una gradual desaceleración, con crecimientos menores a la media histórica.

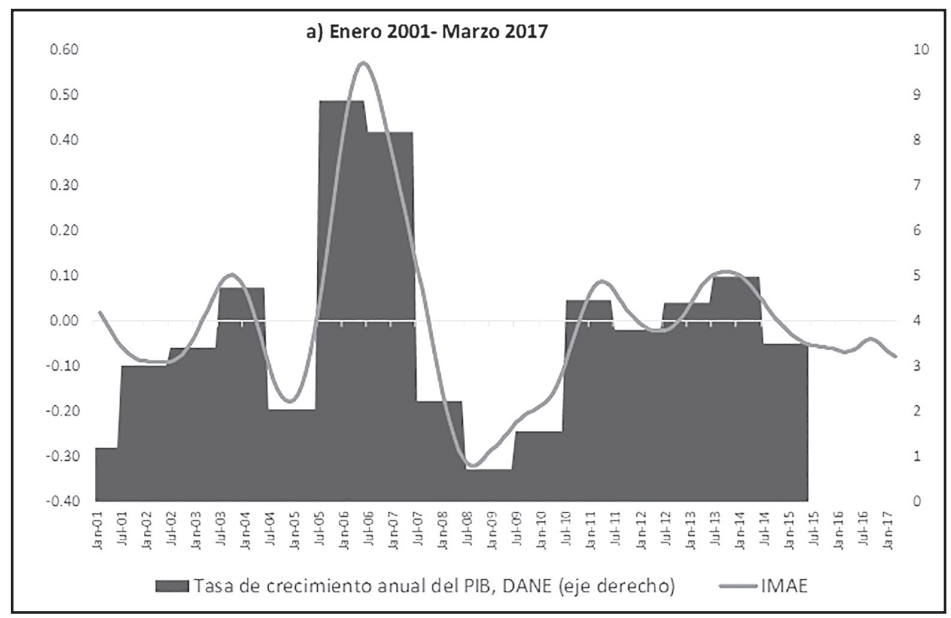

Figura 1. Índice mensual de actividad económica del Valle (IMAE) y crecimiento anual del PIB del Valle.

Los valores del IMAE pueden llevarse además a un modelo de regresión o función de transferencia, donde la variable dependiente sería el PIB mensualizado. De esta forma, el IMAE no solo sería un instrumento para ofrecer información cualitativa sobre el ciclo y las etapas de aceleración o desaceleración, sino que permitiría contar con una estimación cuantitativa sobre el crecimiento del PIB departamental. Se evaluó el poder predictivo del mo- 
delo en una ventana de cinco años. Los resultados de los pronósticos fuera de muestra (out-of-sample) indican resultados satisfactorios, con un error absoluto medio de $0,25 \%$ (ver Tabla 4).
Para el 2015, la proyección del IMAE fue de un crecimiento de $3,9 \%$, mientras que el dato del DANE (segunda revisión) se ubicó en 3,7 \% (un año hacia delante desde 2009).

Tabla 4. Tasa de variación PIB real Valle

\begin{tabular}{|c|c|c|}
\hline & $\begin{array}{c}\text { Pronóstico anual con IMAE } \\
\text { (out-of-sample) }\end{array}$ & Real DANE* \\
\hline 2009 & $0.0 \%$ & $0.7 \%$ \\
\hline 2010 & $1.6 \%$ & $1.6 \%$ \\
\hline 2011 & $4.6 \%$ & $4.5 \%$ \\
\hline 2012 & $3.3 \%$ & $3.8 \%$ \\
\hline 2013 & $4.7 \%$ & $4.6 \%$ \\
\hline 2014 & $4.9 \%$ & $5.0 \%$ \\
\hline
\end{tabular}

Desde el año 2015, en que empiezan las estimaciones del IMAE, este ha venido confirmando la mayor resiliencia de la región al choque que recibió la economía colombiana después de la caída del precio del petróleo. Esto es coherente con una estructura productiva que no depende del sector minero energético, sino que se viene dinamizando con un sector industrial transable que avanza en la sustitución de importaciones y que, gradualmente, impulsa las exportaciones y un gasto de consumo de los hogares, que se ha favorecido con el mayor poder adquisitivo de las remesas después de la depreciación del peso colombiano.

\section{CONCLUSIONES}

Dado el paulatino interés de los agentes por conocer el estado actual de la economía y debido al importante reza- go de las principales estadísticas económicas oficiales disponibles, en este documento se hizo una revisión de la literatura nacional e internacional sobre la construcción de indicadores de actividad económica.

Dentro de los referentes metodológicos de la evidencia actual sobresalió el diseño de índices coincidentes y líderes construidos en Estados Unidos y Europa, especialmente en los bancos centrales, bajo una formalización más estadística, en su mayoría conocidos como modelos factoriales dinámicos (MFD). Esta herramienta toma como punto de partida el MFD de Sargent y Sims (1977), desarrollado posteriormente por Stock y Watson (1991). Este último trabajo ha funcionado como un catalizador para la construcción de indicadores 
mensuales de actividad económica. Sin embargo, la aplicación de tal metodología se ha concentrado en las estadísticas nacionales, pero hay muy pocos ejemplos de indicadores de actividad económica mensuales para una región de un país.

Generalmente, los MFD parten del supuesto de que hay una variable no observada o latente, como el estado de la economía, común a diferentes variables observadas, como las variables mensuales económicas, y que puede ser estimada. Existen varias técnicas para calcular los MFD, y su uso depende de la manera como se extraen los factores y cómo se calcula el componente común. Por eso, una de las contribuciones de este documento fue describir las ventajas y desventajas de los principales métodos para construir indicadores de actividad económica desde los MDF. Así mismo, se presenta la experiencia y método seguido en la construcción del indicador mensual de actividad económica para el Valle del Cauca (IMAE).

Más allá de los temas metodológicos y mirando la utilidad de dicha herramienta instrumental, la experiencia que se ha tenido en el departamento del Valle del Cauca con las primeras estimaciones del IMAE ha sido favorable en cuanto a la comunicación de los resultados y el interés despertado en el sector privado y los medios de comunicación. Si bien el cálculo del indicador constituye para la mayoría de los usuarios finales una especie de "caja negra", eso no ha evitado que este pueda ser interpretado con relativa facilidad e incluso replicado por los medios de comunicación.

La información que reporta el indicador sobre la aceleración o desaceleración de la actividad económica y las proyecciones que ofrece para el crecimiento del PIB del año en curso son accesibles para una amplia gama de empresarios, funcionarios públicos y ciudadanos en general. Este es un punto que tiene a su favor este tipo de aproximaciones, lo cual permite que los esfuerzos en recolección de datos, aplicación de la metodología y la preparación técnica del personal involucrado, tengan un impacto casi inmediato y palpable, pues el indicador resultante tiene grandes posibilidades de convertirse en un referente para los agentes económicos, dada la ausencia de otras mediciones del ciclo con igual frecuencia y prontitud.

\section{AGRADECIMIENTOS}

A la profesora Pilar Poncela, por el seminario Herramientas para la construcción de indicadores de actividad económica, celebrado en marzo de 2014 en la Pontificia Universidad Javeriana de Cali. Este curso motivó la construcción del indicador mensual de actividad económica (IMAE) para el Valle del Cauca y el presente artículo. 


\section{REFERENCIAS}

Alfonso, V., Arango, L., Árias, F., Cangrejo, G. \& Pulido, J. D. (2012). Ciclos de negocios en Colombia: 1975-2011. Banco de la República. Borradores de Economía, (651).

Alonso, J. (2006). Proyectando el producto departamental bruto caucano con un modelo de análisis factorial dinámico. Cali, Colombia: Centro de Investigaciones en Economía y Finanzas (CIENFI), Universidad ICESI.

Angelini, E., Banbura, M. \& Rünstler, G. (2008). Estimating and Forecasting the Euro Area Monthly National Accounts from a Dynamic Factor Model. Journal of Business Cycle Measurement and Analysis, (953).

Arango, L., Árias, F., Flórez, L. A. \& Jalil, M. (2008). Cronología de los ciclos de negocios recientes en Colombia. Lecturas de Economía, (68), 9-37.

Arango, L.E. \& Melo, L.F. (2006). Expansions and Contractions in Brazil, Colombia and Mexico: A View Through Nonlinear Models. Journal of Development Economics, (80), 501-517. Retrieved from https://doi.org/10.1016/j. jdeveco.2005.02.010

Aruoba, B. Diebold, F. \& Scotti, Ch. (2009). Real-Time Measurement of Business Conditions. Journal of Business \& Economic Statistics, 27(4), 41727. Retrieved from https://doi.org/10.1198/jbes.2009.07205

Avella, M. \& Fergusson, L. (2004). El ciclo económico: enfoques e ilustraciones. Los ciclos económicos de Estados Unidos y Colombia. Banco de la República. Borradores de Economía, (284).

Burns, A. F. \& W. C. Mitchell (1946). Measuring Business cycles. In NBER, Studies in Business Cycle. New York: Columbia University Press.

Camacho, M. \& Domenech, R. (2012). MICA-BBVA: A Factor Model of Economic and Financial Indicators for Short-term GDP Forecasting. SERIEs, 3, 475-497. Retrieved from https://doi.org/10.1007/s13209-011-0078-Z

Camacho, M. \& Martínez-Martin, J. (2015). Monitoring the World Business Cycle. Banco de España, Working Paper, (1509). Retrieved from 
https://doi.org/10.1016/j.econmod.2015.09.013, https://doi.org/10.2139/ ssrn.2643954, https://doi.org/10.2139/ssrn.2587001

Camacho, M. \& Pérez-Quirós, G. (2010). Introducing the Euro-STING: Short Term Indicator of Euro Area Growth. Journal of Applied Econometrics, 25(4), 663-694. Retrieved from https://doi.org/10.1002/jae.1174

Camacho, M. Pérez-Quirós, G. \& Poncela, P. (2014). Green shoots and double dips in the euro area: A real time measure. International Journal of Forecasting, 30(3), 520-535. https://doi.org/10.1016/j.ijforecast.2013.01.006

Castro, C. (2003). Yet Another Lagging, Coincident Tan Leading Index for the Colombian Economy. Departamento Nacional de Planeación. Archivos de Economía, (233).

Choi, H. \& Varian, H. (2011). Predicting the Present with Google Trends. The Economic Society of Australia. Economic Record, 87(1).

Doz, C., Giannone, D. \& Reichlin, L. (2011). A Two-Step Estimator for Large Approximate Dynamic Factor Models based on Kalman filtering. Journal of Econometrics, 164(1), 188-205. Retrieved from https://doi.org/10.1016/j. jeconom.2011.02.012

Diebold, F.X. \& Rudebusch, G. (1996). Measuring Business Cycles: A Modern Perspective, Review of Economics and Statistics, 78, 67-77. Retrieved from https://doi.org/10.2307/2109848

Drechsel, K., Giesen, S. \& Lindner, A. (2014). Outperforming IMF Forecasts by the Use of Leading Indicators. Institute for Economic Research. IWH Discussion Papers, (4).

Ferrara, L. \& Marsilli, C. (2014). Nowcasting Global Economic Growth: A Factor-Augmented Mixed-Frequency Approach. Banque de France, (515). retrieved from https://doi.org/10.2139/ssrn.2514218

Forni, M., Hallin, M., Lippi, M. \& Reichlin, L. (2000). The Generalized Factor Model: Identification and Estimation. The Review of Economics and Statistics, 82(4), 540-554. Retrieved from https://doi. org/10.1162/003465300559037 
Forni, M., Hallin, M., Lippi, M. \& Reichlin, L. (2003). The Generalized Dynamic Factor Model: One-sided Estimation and Forecasting. Econpapers. Retrieved from http://econpapers.repec.org/paper/ssalemwps/2003_2f13.htm

Forni, M., Hallin, M., Lippi, M. \& Reichlin, L. (2005). The Generalized Dynamic Factor Model. Journal of the American Statistical Association, 100(471). Retrieved from https://doi.org/10.1198/016214504000002050

Forni, M., Hallin, M., Lippi, M. \& Reichlin, L. (2001). Coincident and Leading Indicators for the Euro Area. The Economic Journal, 111. Retrieved from https://doi.org/10.1111/1468-0297.00620

Giannone, D., Reichlin, L. \& Small, D. (2008). Nowcasting: The Real-Time Informational Content of Macroeconomic Data. Journal of Monetary Economics, 55(4), 665-676. Retrieved from https://doi.org/10.1016/j.jmoneco.2008.05.010

Golinelli, R. \& Parigi, G. (2014). Tracking World Trade and GDP in Real Time. International Journal of Forecasting, 30(4), 847-862. Retrieved from https://doi.org/10.1016/j.ijforecast.2014.01.008

Gómez, A.M., Sarmiento, J.I. \& Fajardo, L. (2016). Advanced Global Indicator of Short and Long Term for the Economy of Cauca 19602014. Apuntes del Cenes, 35(62), 209-244. Retrieved from https://doi. org/10.19053/22565779.5231

Hamilton, J. (1994). Time Series Analysis. Princeton, USA: Princeton University Press.

Kalman, R. E. (1960). A New Approach to Linear Filtering and Prediction Problems. Journal of Fluids Engineering, 82(1). Retrieved from https://doi. org/10.1115/1.3662552

Kamil, H., Pulido, J. \& Torres, J. (2010). El IMACO: un índice mensual líder de la actividad económica de Colombia. Banco de la República. Borradores de Economía, (609).

Kim, M. \& Yoo, J. (1995). New Index of Coincident Indicators: A Multivariate Markov Switching Factor Model Approach. Journal of Monetary Economics, 36, 607-630. Retrieved from https://doi.org/10.1016/03043932(95)01229-X 
Apuntes CENES Volumen 36, Número 64

julio - diciembre 2017. Págs. 79-107

Koopman, S. J., Shephard, N. \& Doornik, J. A. (1999). Statistical Algorithms for Models in State Space Uuing SsfPack 2.2. The Econometrics Journal, 2(1), 107-160. Retrieved from https://doi.org/10.1111/1368-423X.00023

Litterman, R.B. (1983). A Random Walk, Markov Model for the Distribution of Time Series. Journal of Business and Economic Statistics, 1, 169-173. Retrieved from https://doi.org/10.1080/07350015.1983.10509336, https:// doi.org/10.2307/1391858

Marcillo, E. (2013). Un indicador líder para la actividad económica de Colombia. Departamento Nacional de Planeación. Archivos de Economía, (404).

Maurer, M. \& Uribe, M.C. (1996a). El ciclo de referencia de la economía colombiana. Departamento Nacional de Planeación. Archivos de Macroeconomía, (45).

Mejía, L. F., Monsalve, D., Parra., Pulido, S. \& Reyes, A. M. (2013). Indicadores ISAAC: siguiendo la actividad sectorial a partir de Google Trends. Notas Fiscales, Ministerio de Hacienda y Crédito Público, (22).

Melo, L. F., Nieto, F., Posada, C. E., Betancourt, Y. R. \& Barón, J. D. (2001). Un índice coincidente para la actividad económica colombiana. Borradores de Economía, (195).

Melo, L., Nieto, F. \& Ramos, M. (2003). A Leading Index for the Colombian Economic Activity. Banco de la República de Colombia. Borradores de Economía, (243).

Nieto, F. \& Melo, L.F. (2001). About a Coincident Index for the State of the Economy. Documento no publicado.

Poncela, P., Senra, L. \& Sierra, L. (2014). Common Dynamics of Non Energy Commodity Prices and their Relation to Uncertainty. Applied Economics. 46(30), 3724-3735. Retrieved from https://doi.org/10.1080/00036846.20 14.939377

Poncela, P. \& Ruiz, E. (2012). More is not Always Better: Back to the Kalman Filter in Dynamic Factor Models. Madrid: Universidad Carlos III de Madrid, Departamento de Estadística. 
Ripoll, M., Misas, M. \& López, E. (1995). Una descripción del ciclo industrial en Colombia. Banco de la República. Borradores Semanales de Economía, (33).

Rozo, S. (2008). Nuevo enfoque para la construcción de un único indicador líder de la actividad económica colombiana. Ministerio de Hacienda y Crédito Público. Coyuntura Económica, 38(2), 21-62.

Salazar, D. (1996). Gráfico de un sistema de indicadores adelantados y de indicadores coincidentes. M. Maurer, M. Uribe \& J. Birchenall (Eds.), El sistema de indicadores líderes para Colombia (pp. 2-88). Bogotá: DNP.

Schumacher, C. (2007). Forecasting German GDP using Alternative Factor Models based on Large Datasets. Journal of Forecasting, 26(4), 271-302. Retrieved from https://doi.org/10.1002/for.1026

Stock, J. \& Watson, M. (1989). New Indexes of Coincident and Leading Indicators. Mimeo, Cambridge MA: Evanston.

Stock, J. \& Watson, M. (1991). A Probability Model of the Coincident Economic Indicators. In K. Lahiri \& G.H. Moore (eds.). The Leading economic indicators: New approaches and forecasting record (pp. 63-90). Cambridge University Press. Retrieved from https://doi.org/10.1017/ CBO9781139173735.005

Stock, J. H. \& Watson, M. W. (2002). Forecasting Using Principal Components from a Large Number of Predictors. Journal of the American Statistical Association, 97(460), 1167-1179. Retrieved from https://doi. org/10.1198/016214502388618960

Stock, J. H. \& Watson, M. W. (2004). Combination Forecasts of Output Growth in a Seven-Country Data Set. Journal of Forecasting, 23(6), 405-430. Retrieved from https://doi.org/10.1002/for.928

Stock, J. H. \& Watson, M. (2011). Dynamic Factor Models. Oxford Handbook on Economic Forecasting. Retrieved from https://doi.org/10.1093/oxford$\mathrm{hb} / 9780195398649.013 .0003$

Vidal, P., Sierra, L., Sanabria, J. \& Collazos, J. (2015). Indicador mensual de actividad económica (IMAE) para el Valle del Cauca. Banco de la República. Borradores de Economía, (900). 
Apuntes CENES Volumen 36, Número 64

julio - diciembre 2017. Págs. 79-107

\section{APÉNDICES}

Tabla 1. Variables seleccionadas con la actividad económica

Tránsito de vehículos de carga por los peajes de la malla vial del Valle del Cauca y Cauca, MVVCC: el principal medio de transporte para movilizar la producción industrial y sus insumos en el Valle del Cauca se hace a través del transporte de carga terrestre, para lo cual se realiza el conteo de vehículos pesados por los peajes de la región, tanto al ingreso como salida. En el departamento, según cifras del PIB departamental publicado por el DANE, más del $50 \%$ de la rama de transporte, almacenamiento y comunicaciones corresponde a transporte terrestre, lo cual confirma que una parte importante de la carga de la región es movilizada por carretera. De esta forma, el volumen de vehículos de carga que transitan por el departamento se convierte en una variable para observar directamente la evolución de la actividad de transporte e indirectamente la de la actividad industrial, comercial y de servicios, en forma contemporánea. El mayor tránsito de camiones y tractocamiones por los peajes locales se relaciona directamente con cambios positivos en la actividad económica del departamento, por los encadenamientos existentes con otras actividades, como servicios asociados al transporte, el empleo, el comercio y la industria, entre los más directos.

Cartera bruta: el crédito como medio de pago para las transacciones de bienes y servicios es una fuente para financiar principalmente el consumo en los hogares. Los recursos se utilizan para apalancar el comercio de vehículos, vivienda, educación, etc. y en las empresas facilita la ampliación de la producción, para impulsar el crecimiento de la economía del departamento. Si bien el seguimiento a la cartera bruta se constituye en un indicador de la actividad de servicios, en particular de la evolución de la actividad financiera, también indirectamente da cuenta de las condiciones macroeconómicas, como la ocupación y formalización en el mercado laboral, condiciones necesarias para el acceso al crédito.

Índice de empaques: en términos generales, todo producto manufacturado con destino al consumidor final requiere de algún tipo de embalaje. Las industrias al momento de recibir los pedidos de mercancías para el periodo siguiente, solicitan con anticipación los empaques al proveedor respectivo, que por lo general se localiza en el Valle del Cauca al concentrar más del $90 \%$ de la oferta de este insumo del proceso productivo. Cuando una empresa 
manufacturera prevé incrementar su producción demanda previamente mayor cantidad de empaques. A su vez, cuando las empresas de empaques aumentan su producción es porque se ha percibido un impulso al crecimiento del sector fabril y del comercio, líderes en la actividad económica en general.

Índice de confianza del consumidor (ICC): es un índice publicado por FEDESARROLLO que mide la confianza del consumidor sobre su percepción del estado actual y futuro de la economía de Cali. Por esta razón, se convierte en un buen validador sobre el comportamiento que tiene y tendrá la economía.

Índice de condiciones económicas (ICE): es un índice publicado por FEDESARROLLO que mide las condiciones económicas actuales de los hogares desde su disponibilidad para comprar bienes durables y semidurables. En la medida en que los hogares se encuentren en una bonanza económica, así mismo incrementan el consumo por estos bienes de manera significativa.

Tabla 2. Descripción del tratamiento utilizado a las series que componen el IMAE

\begin{tabular}{|c|l|c|c|c|c|}
\hline & \multicolumn{1}{|c|}{ Descripción } & Fuente & Estacionalidad & $\begin{array}{c}\text { Grado de } \\
\text { integración }\end{array}$ & $\begin{array}{c}\text { Transforma- } \\
\text { ción }\end{array}$ \\
\hline 1 & Caña molida & Asocaña & Sí & $\mathrm{I}(0)$ & S,E,D \\
\hline 2 & $\begin{array}{l}\text { Venta de vehículos } \\
\text { nuevos }\end{array}$ & Asonac & Sí & $\mathrm{I}(1)$ & S,E,L,D \\
\hline 3 & $\begin{array}{l}\text { Despachos de } \\
\text { cemento }\end{array}$ & DANE & Sí & $\mathrm{I}(1)$ & S,E,L,D \\
\hline 4 & $\begin{array}{l}\text { Índice de } \\
\text { Producción } \\
\text { Industrial Regional, } \\
\text { IPIR }\end{array}$ & $\begin{array}{l}\text { Banco Repú- } \\
\text { blica Cali }\end{array}$ & Sí & $\mathrm{I}(1)$ & S,E,L,D \\
\hline 5 & $\begin{array}{l}\text { Exportaciones } \\
\text { US\$FOB - Valle }\end{array}$ & DANE-DIAN & Sí & $\mathrm{I}(1)$ & S,E,L,De,D \\
\hline 6 & $\begin{array}{l}\text { Importaciones } \\
\text { US\$CIF - Valle }\end{array}$ & DANE-DIAN & Sí & $\mathrm{I}(1)$ & S,E,L, De,D \\
\hline
\end{tabular}


Apuntes CENES Volumen 36, Número 64

julio - diciembre 2017. Págs. 79-107

\begin{tabular}{|c|c|c|c|c|c|}
\hline 7 & $\begin{array}{l}\text { Demanda de } \\
\text { energía eléctrica no } \\
\text { residencial -Valle }\end{array}$ & $\begin{array}{l}\text { X.M. Filial de } \\
\text { ISA }\end{array}$ & Sí & $\mathrm{I}(1)$ & S,E,L,D \\
\hline 8 & $\begin{array}{l}\text { Tránsito de vehícu- } \\
\text { los de carga por los } \\
\text { peajes de la MVVCC }\end{array}$ & $\begin{array}{c}\text { Agencia } \\
\text { Nacional de } \\
\text { Infraestruc- } \\
\text { tura }\end{array}$ & Sí & $\mathrm{I}(1)$ & S,E,L,D \\
\hline 9 & Cartera Bruta & $\begin{array}{l}\text { Superfinan- } \\
\text { ciera }\end{array}$ & Sí & $\mathrm{I}(1)$ & S,E,L,D \\
\hline 10 & Índice de Empaques & $\begin{array}{l}\text { Banco Repú- } \\
\text { blica Cali }\end{array}$ & Sí & $\mathrm{I}(1)$ & S,E,L,D \\
\hline 11 & $\begin{array}{l}\text { Índice de Confianza } \\
\text { del Consumidor } \\
\text { (ICC) - Cali }\end{array}$ & Fedesarrollo & Sí & $\mathrm{I}(1)$ & S,E,D \\
\hline 12 & $\begin{array}{l}\text { Índice de } \\
\text { Condiciones } \\
\text { Económicas (ICE) } \\
\text { - Cali }\end{array}$ & Fedesarrollo & Sí & $\mathrm{I}(1)$ & S,E,D \\
\hline
\end{tabular}

S: serie desestacionalizada.

E: serie estandarizada.

L: serie transformada con el logaritmo natural.

De: serie deflactada con el IPP de Estados Unidos.

D: serie transformada con la primera diferencia. 
